\title{
Long-term disease stabilization following treatment with erlotinib in heavily pretreated patients with wild-type epidermal growth factor receptor non-small-cell lung carcinoma: Two case reports
}

\author{
MINAS SAKELLAKIS ${ }^{1}$, ANGELOS KOUTRAS ${ }^{1}$, MARIA PITTAKA ${ }^{2}$, EFSTATHIOS TSITSOPOULOS ${ }^{3}$, \\ FOTINI KALOFONOU ${ }^{1}$ and HARALABOS P. KALOFONOS ${ }^{1}$ \\ ${ }^{1}$ Division of Oncology, Department of Medicine; ${ }^{2}$ Department of Radiation Oncology, University Hospital, \\ Patras Medical School, 26504 Patras; ${ }^{3}$ Molecular Biology Diagnostic Center 'Bioiatriki', 11526 Athens, Greece
}

Received May 30, 2016; Accepted July 25, 2016

DOI: $10.3892 / \mathrm{mco} .2016 .1036$

\begin{abstract}
Lung adenocarcinomas carrying epidermal growth factor receptor (EGFR) mutations have been identified as a unique group of entities that depend on EGFR for their proliferation and metastasis. The introduction of reversible EGFR tyrosine kinase inhibitors, such as erlotinib, has significantly affected the management of metastatic disease in this subset of patients. Interestingly, although erlotinib is highly effective in patients with EGFR mutations, it may occasionally prove useful, even in the absence of mutations. We herein present the course of two heavily pretreated patients who achieved remarkable disease stabilization over several years, despite harbouring no EGFR mutations. Our cases underscore the fact that further research is required to identify which subset of patients will benefit the most from this treatment, as a substantial minority may present with favourable outcomes.
\end{abstract}

\section{Introduction}

Lung cancer has the highest mortality rate worldwide, with a 5 -year survival of only $15 \%$ (1). Non-small-cell lung cancer (NSCLC) is considered to be the most frequent type of lung cancer, accounting for $85 \%$ of the total number of cases (1). Unfortunately, the disease is usually progressed at diagnosis and locoregional strategies are no longer considered as therapeutic options. Until recently, cytotoxic chemotherapy was the only treatment option for advanced disease, with a median overall survival of 12 months (1).

Correspondence to: Dr Minas Sakellakis, Division of Oncology, Department of Medicine, University Hospital, Patras Medical School, Riou-Patron Street, Rion, 26504 Patras, Greece

E-mail: doctorsakellakis@gmail.com

Key words: erlotinib, tyrosine kinases, stabilization, heavily pretreated
NSCLC comprises several subtypes in terms of histological and morphological characteristics, exhibiting significant genetic variability (2). Lung adenocarcinomas carrying epidermal growth factor receptor (EGFR) mutations were recently identified as a unique group of entities, based on common etiopathogenetic characteristics and unique tumor biology; importantly, they all depend on EGFR for their proliferation and metastasis (2). The introduction of reversible EGFR tyrosine kinase inhibitors (TKIs), such as erlotinib, has changed the management of metastatic disease in this subset of patients (3).

Interestingly, although erlotinib is highly effective in patients with EGFR mutations, it may occasionally prove useful, even in the absence of mutations. For example, Shepherd et al concluded that, in pretreated patients, erlotinib achieved a response rate of $8.9 \%$ and the median duration of the response was 7.9 months, irrespective of their mutation status (4). The progression-free and overall survival were 2.2 and 6.7 months, respectively, which were significantly superior to those in patients receiving best supportive care (4). The exact mechanism underlying the effectiveness of erlotinib in this setting remains unknown, and every effort to explain this benefit is highly speculative.

We herein present the cases of 2 patients with advanced disease who were treated with oral erlotinib and achieved remarkable disease stabilization for several years, despite harbouring no EGFR mutations (EGFR wild-type).

Case 1. A 54 year-old male patient, who was a former smoker, was referred to the Division of Oncology, Department of Internal Medicine, University Hospital of Patras Medical School (Patras, Greece) with lung adenocarcinoma in February, 2005. The diagnosis was made following bronchoscopy and cytological material examination, obtained after a thoracic computed tomography (CT) scan revealed a $3-\mathrm{cm}$ mass in the upper segment of the right lower lobe, with enlarged ipsilateral hilar and mediastinal lymph nodes. Imaging also revealed enlarged contralateral (left) cervical and supraclavicular lymph nodes. Additionally, a brain CT scan revealed a lesion in the left temporal lobe, surrounded by significant edema. The patient was referred for brain radiation therapy 
and his neurological signs improved. Following radiotherapy, the patient received 6 cycles of paclitaxel/gemcitabine (from May to July, 2005), followed by radiotherapy to the lung and mediastinum. The patient was then placed on a surveillance program. After 3 months, follow-up imaging revealed a newly enlarged ipsilateral supraclavicular lymph node. Although second-line chemotherapy treatment with 6 cycles of cisplatin/ pemetrexed was administered to the patient, there was disease progression to the right lung. Thus, the patient received third-line chemotherapy with 6 cycles of docetaxel (from April to August, 2006). Restaging showed that the patient achieved partial remission, while the cervical and supraclavicular lymph nodes were no longer palpable on physical examination (Fig. 1A). A chest CT scan, performed in January, 2009, revealed disease progression and the patient was initiated on fourth-line treatment with erlotinib (Tarceva; Roche, Basel, Switzerland), $150 \mathrm{mg}$ per os, once daily. There were no signs of further progression based on follow-up imaging, indicating stable disease. In December, 2015, almost 7 years after the initiation of therapy with erlotinib, the patient remained in a good overall condition, without clinical or radiological signs of progression (Fig. 1B). The cytological material was retrospectively analyzed and the tumor was found to be EGFR wild-type, as reverse transcription polymerase chain reaction analysis revealed no mutations in exons 18, 19, 20 or 21 of the EGFR gene.

Case 2. A 61-year-old male patient, who was a smoker, was diagnosed with undifferentiated NSCLC, according to the histological report, in March, 2006. As shown in Fig. 2A, the tumor, sized $12 \times 9.8 \times 8.2 \mathrm{~cm}$, was located in the middle lobe of the right lung and it was infiltrating the lower pulmonary vein (T4 based on the TNM staging system) (5). The specimen was tested for EGFR mutations in exons 18, 19, 20 and 21, and the results revealed that the tumor was wild-type. The patient was not eligible for lung resection due to his physical and medical condition.

In May, 2006, a combination of cisplatin and etoposide was administered to the patient, with the addition of radiation therapy, until July, 2006. A follow-up performed in November, 2006 revealed that the disease had progressed, as a new mediastinal mass was found, sized $4.5 \times 3.5 \mathrm{~cm}$, infiltrating the esophageal wall. One month later, while on chemotherapy with docetaxel, the patient was diagnosed with disease progression in the thorax and was placed on a new chemotherapy scheme with pemetrexed. Subsequently, the patient received 9 cycles prior to being diagnosed with a new pleural effusion of neoplastic origin in October, 2007.

The patient therefore received oral erlotinib (150 mg once per day) and achieved stable disease for $>5.5$ years (from October, 2007 to May, 2013) (Fig. 2B). However, in May 2013, the patient noticed an enlarged left cervical lymph node. A biopsy of the lesion revealed the histological characteristics of squamous cell lung carcinoma (EGFR wild-type).

Subsequently, the patient received further treatment with different chemotherapeutic schemes, including carboplatin/ gemcitabine, carboplatin/paclitaxel and vinorelbine. In April, 2014 , the condition of the patient deteriorated significantly and he developed disease progression, eventually leading to death after a brief period of time.

\section{Discussion}

Tyrosine kinases (TKs) control critical pathways that regulate important cellular activities (6). Once overexpressed or mutated, TKs play a significant role in the pathogenesis and development of malignancies. When a tumor is dependent on such a mechanism, specific molecules targeting these pathways may be considerably effective (6).

However, erlotinib does not have long-lasting effects, since all tumors inevitably develop resistance after a median of 6-12 months (1). Half of the cases will carry a number of second-site mutations, which are able to alter the binding of the drug to the EGFR-specific domain. The most common event is an acquired T790M missence mutation (referred to as gatekeeper mutation) that accounts for $50-60 \%$ of the acquired resistance (1). Amplification of MET tyrosine kinase is another mechanism that accounts for $22 \%$ of the cases and occurs with or without a concurrent T790M mutation (1). Another known mechanism includes transformation to small-cell lung cancer phenotypes (14\%) (1).

The response rates are particularly high in patients with EGFR mutations who are administered EGFR inhibitors as first-line therapy (6). In selected patients with mutation positivity, the response rates with first-line treatment with EGFR inhibitors may be as high as $75 \%$ (1). However, when administered as second- or third-line treatment, the levels of EGFR mutation and tumor sensitivity were not found to be strongly correlated, indicating a possible non-EGFR-related effect in this subgroup of patients $(6,7)$.

Apart from the EGFR status, other factors have been suggested to be predictive biomarkers of a positive outcome after receiving an EGFR inhibitor, including smoking history, female gender, Japanese ethnicity, adenocarcinoma with bronchoalveolar characteristics and the development of a skin rash $(7,8)$. Both patients described herein were Caucasian. Moreover, we were unable to define the presence of bronchoalveolar characteristics in the first patient, whereas the tumor of our second patient appeared to be of squamous cell histology. Furthermore, our patients were male and smokers, suggesting that the effect of erlotinib was independent of the classical risk factors. Of note, during the entire course of his treatment, the first patient did not complain of a severe skin rash; however, the second patient developed a grade 2 acne-like rash. Both patients achieved long-term disease stabilization and survival, despite the fact that they did not achieve complete or partial response. Determining the EGFR status in patients with squamous cell lung carcinoma remains controversial. However, our second case underscores that a subset of patients may benefit from EGFR inhibitors, with some patients achieving durable disease stabilization.

A retrospective long-term survival study of patients receiving therapy with erlotinib performed in Germany, evaluated the characteristics of patients (irrespective of the EGFR status) who survived over 12 months, and they matched those of the present cases (9). However, in the German study, only $25 \%$ of long-term survivors reached 24 months while on treatment (9).

What caused our patients to exceed the 60-70-month time frame with progression-free survival remains unknown, and every effort to explain why certain patients achieve significant benefits remains highly speculative. Erlotinib is a molecule that specifically targets EGFR TKs, as it binds reversibly to 

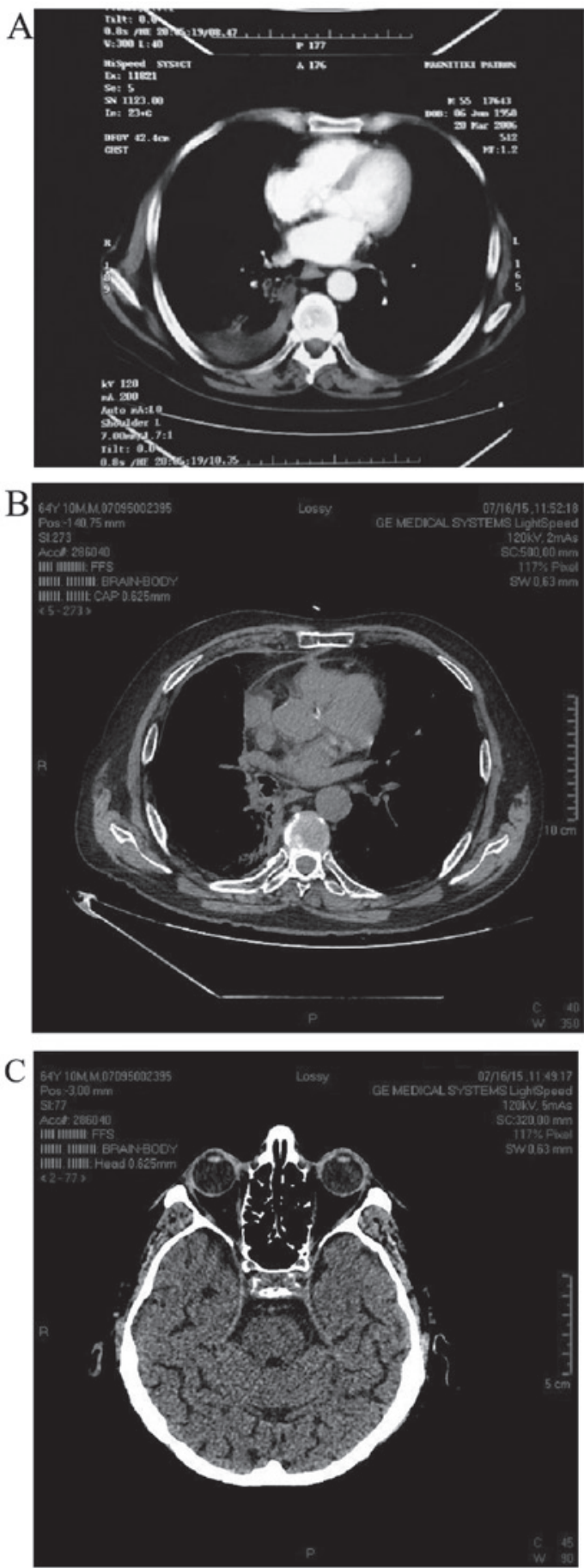

Figure 1. Case 1. (A) 21/03/2006: Axial chest computed tomography (CT) scan showing a $3-\mathrm{cm}$ mass in the lower right lobe in close proximity to the pleura, with a pleural effusion in the right hemithorax. (B) 16/07/2015: The patient presented with stable disease and improved radiological imaging findings, with no signs of local or distant recurrence. (C) There were no signs of relapse on a follow-up CT scan 9 years after whole-brain irradiation.

the adenosine triphosphate binding site of the receptor. Thus, signaling cascades are not initiated and EGFR-dependent tumors are inhibited. However, our patients had no detectable mutations, which means that there are alternative mechanisms underlying the action of erlotinib. A possible explanation is that our patients possibly had an amplification in the gene responsible for EGFR activation, thus having an overactive $\mathrm{TK}$, without harboring any mutations.
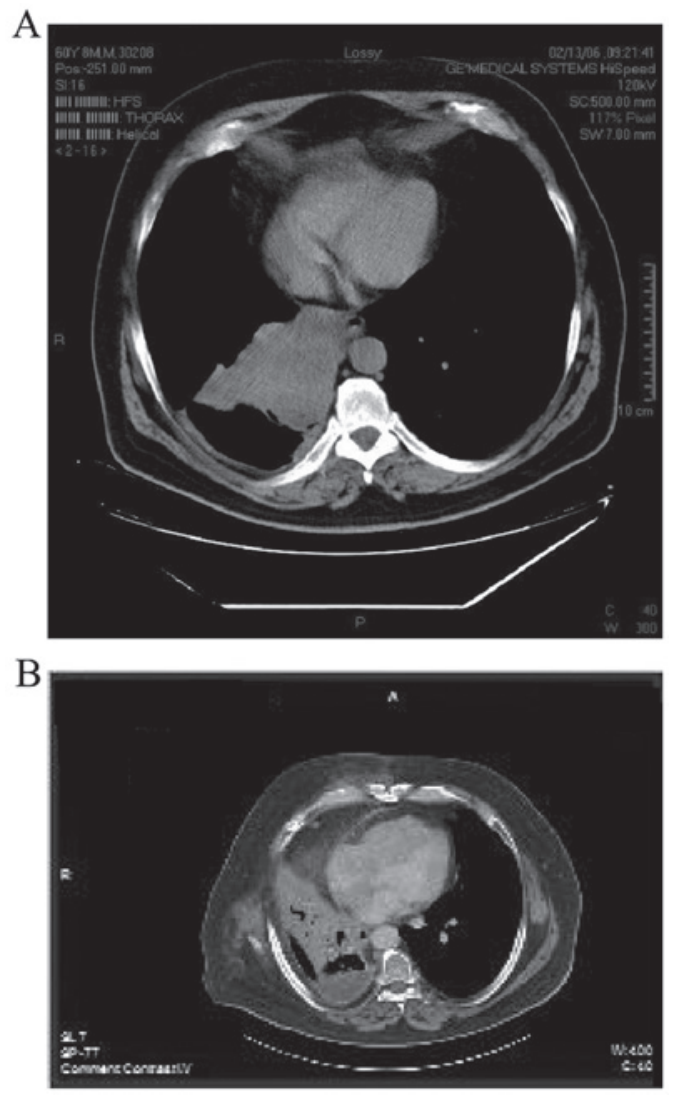

Figure 2. Case 2. (A) 13/02/2006: Axial image showing a large tumor $(12 \times 9.8 \times 8.2 \mathrm{~cm})$ occupying the majority of the lower right lobe. The tumor appeared to be in close contact with the pericardium and mediastinum, strangling the main bronchus of the median and lower lobe. (B) 30/07/2012: Mild chronic pleural effusion. Additional elements with soft tissue density are noted in the right pulmonary hilar region, with narrowing of the right main bronchus. Atelectasis of the right lower lobe was also observed. The imaging findings were stable compared with the previous follow-up.

From a clinical perspective, our patients, as well as one other case previously reported in the literature that exceeded 5 years of disease stabilization, exhibited no spread of the disease to the liver, bones, or adrenal glands, despite the long course of their disease (10). By contrast, they were mainly characterized by bulky nodal disease, pointing towards tumors with a predisposition to lymphatic spread, and a relative inability for hematogenous spread. Lymphatic spread is unique, as neovascularization is not an essential prerequisite (11). It was recently demonstrated that, compared with hematogenous spread, lymphatic spread is independent of epithelial-to-mesenchymal transition (EMT) in the primary tumor (12). This is particularly important, as it indicates that, in our patients, the tumors may have had an intrinsic defect in the EMT pathway, predisposing to lymphatic rather than hematogenous spread. Moreover, the activity of erlotinib has been shown to be correlated with EMT status via interfering with the ILK-PINCH-Parvin complex (13). Therefore, we may hypothesize that erlotinib may have affected the EMT pathway in our patients.

In conclusion, TKI inhibitors may occasionally be associated with impressive long-term disease stabilization, irrespectively of the EGFR status. Unfortunately, these patients constitute a substantial minority. Further research is required to identify the subset of patients that will benefit the most from this treatment. 
Written informed consent was obtained from the patients for the publication of the case reports and any accompanying images.

\section{References}

1. Nelson V, Ziehr J, Agulnik M and Johnson M: Afatinib: Emerging next-generation tyrosine kinase inhibitor for NSCLC. Onco Targets Ther 6: 135-143, 2013.

2. Köhler J and Schuler M: Afatinib, erlotinib and gefitinib in the first-line therapy of egfr mutation-positive lung adenocarcinoma: A review. Onkologie 36: 510-518, 2013.

3. Health Quality Ontario: Epidermal Growth Factor Receptor Mutation (EGFR) testing for prediction of response to EGFR-Targeting Tyrosine Kinase Inhibitor (TKI) drugs in patients with advanced non-small-cell lung cancer: An evidence-based analysis. Ont Health Technol Assess Ser 10: $1-48,2010$.

4. Shepherd FA, Rodrigues Pereira J, Ciuleanu T, Tan EH, Hirsh V, Thongprasert S, Campos D, Maoleekoonpiroj S, Smylie M, Martins R, et al: Erlotinib in previously treated non-small-cell lung cancer. N Engl J Med 353: 123-132, 2005.

5. Goldstraw P, editor. Staging Manual in Thoracic Oncology. Editorial Rx Press, Orange Park, FL, 2009.

6. Pao W, Miller V, Zakowski M, Doherty J, Politi K, Sarkaria I, Singh B, Heelan R, Rusch V, Fulton L, et al: EGF receptor gene mutations are common in lung cancers from 'never smokers' and are associated with sensitivity of tumors to gefitinib and erlotinib. Proc Natl Acad Sci USA 101: 13306-13311, 2004.
7. Karam I and Melosky B: Response to second-line erlotinib in an EGFR mutation-negative patient with non-small-cell lung cancer: Make no assumptions. Curr Oncol 19: 42-46, 2012.

8. Dudek AZ, Kmak KL, Koopmeiners J and Keshtgarpour M Skin rash and bronchoalveolar histology correlates with clinical benefit in patients treated with gefitinib as a therapy for previously treated advanced or metastatic non-small cell lung cancer. Lung Cancer 51: 89-96, 2006

9. Schumann C, Heigener D, Dittrich I, Abenhardt W, Dederke B, Duell T, et al: Long term benefit from erlotinib treatment is independent of prognostic factors and therapeutic response (abstract 9146). Eur J Cancer 7: 1, 2009.

10. Polychronidou G and Papakotoulas P: Long-term treatment with erlotinib for EGFR wild-type non-small cell lung cancer: A case report. Case Rep Oncol 6: 189-196, 2013.

11. Jeong HS, Jones D, Liao S, Wattson DA, Cui CH, Duda DG, Willett CG, Jain RK and Padera TP: Investigation of the lack of angiogenesis in the formation of lymph node metastases. J Natl Cancer Inst 107: pii: djv155, 2015.

12. Markiewicz A, Książkiewicz M, Seroczyńska B, Skokowski J, Szade J, Welnicka-Jaskiewicz M, Wełnicka-Jaśkiewicz M and Zaczek AJ: Heterogeneity of mesenchymal markers expression-molecular profiles of cancer cells disseminated by lymphatic and hematogenous routes in breast cancer. Cancers 5: 1485-1503, 2013.

13. Augustin A, Lamerz J, Meistermann H, Colling S, Scheiblich S, Hermann JC, Duchateau-Nguyen G, Tzouros M, Avila DW, Langen $\mathrm{H}$, et al: Quantitative chemical proteomics profiling differentiates erlotinib from gefitinib in EGFR wild-type non-small cell lung carcinoma cell lines. Mol Cancer Ther 12: 520-529, 2013. 